\title{
An experience of femtosecond laser assisted cataract surgery in advanced brown cataracts in a tertiary Eye Center of Europe
}

\author{
Bastola , $^{1 *}$ Dick $\mathrm{BH}^{2}$ \\ ${ }^{I}$ Department of Ophthalmology, Nepalgunj Medical College and Teaching Hospital, Nepalgunj, Nepal, \\ ${ }^{2}$ Professor, Director, Ruhr University Eye Hospital, Center for Vision Science and research, \\ Department of Ophthalmology, 44892 Bochum, Germany
}

\begin{abstract}
*Corresponding Author:
Dr. Pradeep Bastola,

MD, Ophthalmologist,

Assistant Professor, Department of Ophthalmology

Nepalgunj Medical College and Teaching Hospital,

Email: drbastola15@gmail.com

Citation

Bastola P, Dick BH. An experience of femtosecond laser assisted cataract surgery in advanced brown cataracts in a tertiary Eye Center of Europe. Nepal Journal of Medical Sciences 2013;2(2):114-8.
\end{abstract}

\section{Background:}

Cataract surgery is the most widely performed intraocular procedure. As a result, techniques are under constant review

\begin{abstract}
Background: To assess the results of femtosecond laser use in harder brown cataracts and share the first time experience.

Methods: Thirty eyes of thirty patients were included in this hospital based cross sectional descriptive study. The study was carried out from April, 2012 to July, 2012 in University of Bochum, Vision research center, Germany. All surgeries were performed by a single surgeon (Burkhard Dick) using the Catalys femtosecond cataract laser (Optimedica; Santa Clara, California) and a standardized "stop and chop" phaco technique with the Stellaris (Bausch + Lomb; Rochester, New York) phaco machine in venturi fluidics mode. Relevant data was entered in a proforma designed for the study and interpreted.
\end{abstract}

Results: In the study, $19(63.3 \%)$ of the patients were females followed by males. The mean age of the patients was 74.83 years (Std. deviation 6.56). According to lenticular opacification classification system III (LOCS III); mean nuclear sclerosis was 3.9 (Std. deviation 0.80), mean absolute phaco time (APT) was 0.19 seconds (Std. deviation 0.24 seconds) mean effective phaco time (EPT) was 0.02 seconds (Std. deviation 0.04) and mean phaco power (MPP) used was 9.26\% (Std. deviation 8.24). Amongst complications; remnant of anterior capsular tag and posterior capsular rupture (PCR) was noted in two patients respectively.

Conclusion: This study demonstrated that, with further innovation and optimization of technique and instruments femtosecond laser-assisted cataract surgery will become phacoless ("aphaco") for most cataracts, whereas dense cataracts may still require the "pauci-phaco" (reduced phaco energy) technique.

Keywords: hard brown cataracts; femtosecond laser; mean effective phaco time; mean phaco power

and are undergoing evolution which has brought about a revolution. ${ }^{1,2}$ The techniques of cataract surgery has evolved from large-incision extracapsular extraction to microincision surgeries. Patient expectations also have changed, with an 
increasing emphasis on precise refractive as well as visual and safety outcomes. The introduction of femtosecond lasers to cataract surgery represents a significant advancement in cataract technology. ${ }^{3}$ Femtosecond lasers have been used successfully in ophthalmic surgery since 2001 . $^{4-8}$

Since 2001, several femtosecond laser systems have been introduced and more than 2 million ophthalmic procedures have been performed with these lasers. With their computercontrolled optical delivery systems, femtosecond laser machines can produce precise surgical incisions without collateral damage to surrounding tissues. The femtosecond laser has received food and drug administration (FDA) clearance for use in cataract surgery and is being used widely all around the globe at present. ${ }^{9}$

Capsulorrhexis for cataract surgery has been a manual procedure even now in many developing countries. But with the advent of femtosecond laser and its use in cataract surgery a predictable sized and centered capsulorrhexis has been possible due to laser tissue interaction known as photodisruption. ${ }^{10}$

Multiple research studies have shown that manual capsulorrhexis is known to be the most technically difficult part of cataract surgery for trainees more so over in hard/ denser cataracts. It leads to tears in $0.8 \%$ of cases. The femtosecond laser delivers a more circular, stronger, precisely planned and executed capsulorrhexis, which could afford more control over capsulotomy unpredictability and offer more accurate refractive outcomes. ${ }^{11,12}$

With so much going on; around world; at present with regard to use of femtosecond laser in cataract surgery starting from creating corneal flaps, arcuate corneal incision, clear corneal incisions, performing anterior capsulotomies, lens fragmentation, posterior capsulotomies etc. Still the published results of femtosecond laser cataract surgeries are few and there has not been a single study till date about the use of femtosecond laser in harder difficult brown cataracts henceforth, a study on femtosecond laser assisted surgeries for harder brown cataracts was worthy. Hence, this study was carried out in patients having harder brown cataracts undergoing femtosecond laser assisted cataract surgery with regard to all the measures of a normal femtosecond laser assisted phacoemulsification cataract surgery in a tertiary eye center of Europe. This is probably the first experience of such kind of study universally.

\section{Methods:}

This study included 30 suitable patients willing to undergo femtosecond laser cataract surgeries having a lenticular opacification classification system III (LOCS III) ${ }^{13}$ nuclear sclerosis grading more than three. The study was carried out in Department of Ophthalmology, Ruhr University Eye Hospital, Bochum, Germany, between April, 2012 and July, 2012. The surgeries were performed by a single surgeon (HB Dick) who holds a distinct reputation of prior extensive experience with the use of the femtosecond laser in cataract surgeries, Refractive surgeries as well as other types Ophthalmic surgeries.

The study adhered to the tenets of the Declaration of Helsinki. Ethical approval was obtained from the local human research ethics committee. The study was a, hospital based, non-randomized, cross sectional, descriptive clinical evaluation. Eligible subjects were examined before surgery to establish a baseline for their ocular condition.

An in-depth discussion about the risks and benefits of the procedure was provided to the patients having denser brown cataracts, followed by obtaining informed written consent. All patients underwent a detailed clinical assessment. The preoperative evaluations included measurement of uncorrected and corrected distance visual acuity, manifest refraction, and corrected near visual acuity. Slit-lamp biomicroscopic examination, tonometry was carried out in all cases. Other investigations included measurement of axial length and biometry (IOL master, Carl Zeiss, Germany), pachymetry, corneal topography, densitometry, specular microscopy, and optical coherence tomography (OCT) besides; pentacam study was also done. Routine blood investigations to rule out Diabetes mellitus, lipid disorders and seeing the hematocrit values were followed as per the center's protocol. In all cases intravenous line (IV line) was opened before taking the patients to the operation theatre.

Patients with glaucoma, corneal opacity, hypotony, poorly dilating pupils $(<6 \mathrm{~mm})$, lens or zonular instability, blood, or any other material in the anterior chamber were excluded from femtosecond cataract surgery. According to the hospital's standard cataract surgery protocol, patients instilled topical eye drops in the eyes prior to surgery.

The OptiMedica Catalys laser system was used to perform all the femtosecond cataract procedures. The Catalys is a next generation laser cataract surgery system that brings unparalleled precision and accuracy and a markedly streamlined workflow to the laser cataract procedure. The system combines a femtosecond laser, integrated 3D Optical Coherence Tomography (OCT) imaging and OptiMedica's 
breakthrough pattern scanning technology in an ergonomic, easy-to-use system that delivers a precise, customized procedure for patients. Distinguishing features include a Liquid Optics ${ }^{\mathrm{TM}}$ Interface that ensures stable system-patient attachment and optimizes the optical path to the patient's eye, and a proprietary Integral Guidance ${ }^{\mathrm{TM}}$ system that ensures the femtosecond laser pulses are delivered safely and precisely to the intended location. In addition, Catalys features an easy-to-use and elegant graphical user interface designed to simplify the planning process and minimize the time the patient is under the dock. ${ }^{14}$

All the cases were given intravenous midazolam (1mg) injection as a precaution to avoid anxiety and for bit of sedation. All surgeries were performed under topical/local anesthesia with $0.4 \%$ oxybuprocaine hydrochloride/4\% lignocaine or peribulbar anesthesia using $2 \%$ lignocaine injection mixed with diluted adrenaline and hyalse. Pupillary dilation was achieved before surgery with $1 \%$ tropicamide or use of intracameral adrenaline on the operation table

The initial steps for the procedures included programming of the laser system, incorporating the patient details and data in the system, setting up the laser system for anterior capsulotomy, lens fragmentation, primary incision, and secondary incision patterns when planned. After all pattern selections and parameter choices were complete, the system was made ready to dock to the patient eye. (Photograph 1)

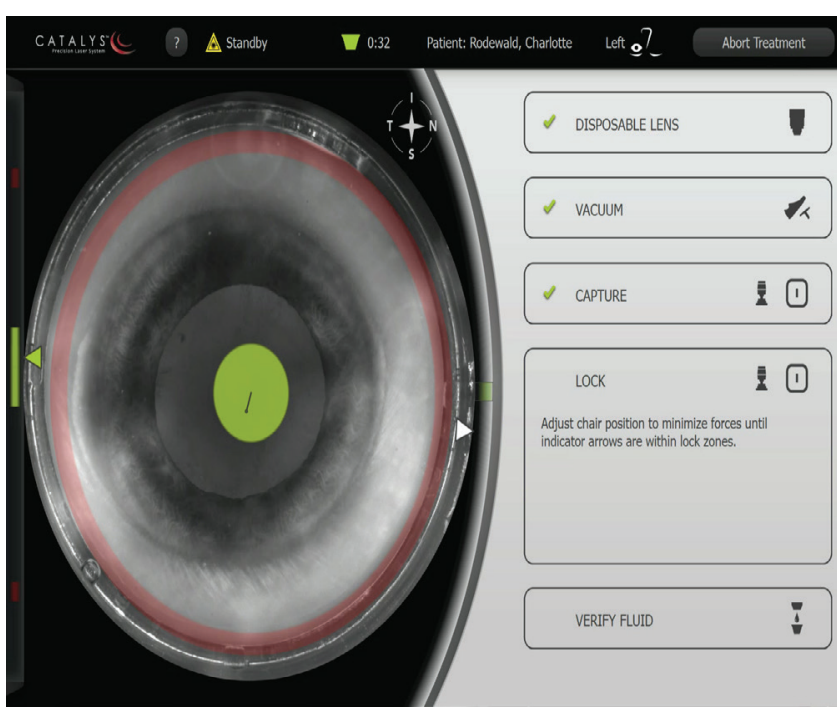

Photograph 1, showing a docked eye just before application of femtosecond laser.

Post successful docking laser procedure was carried out in all cases femtosecond laser was used to perform continuous curvilinear capsulorrhexis (CCC) and lens fragmentation, (Photograph 2) while in few cases primary and secondary clear corneal incisions (CCI) were also performed.

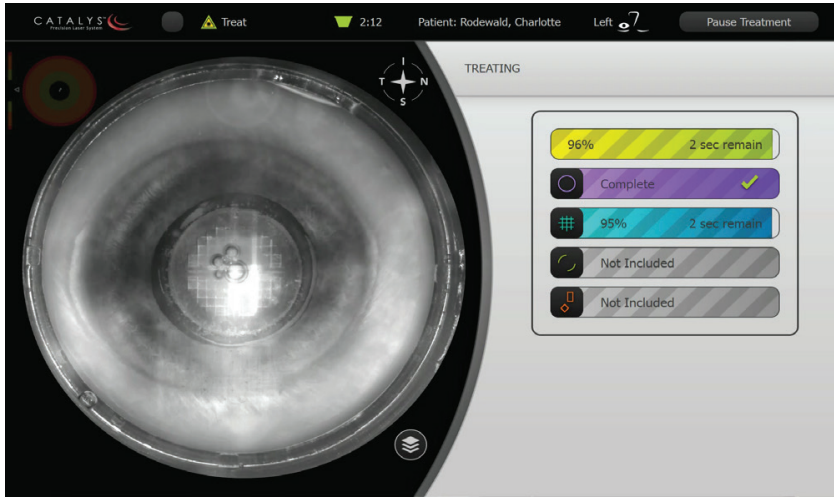

Photograph 2 showing femtosecond laser treatment to create a continuous curvilinear capsulorrhexis, with almost done lens fragmentation, however arcuate and clear corneal incisions were not included in this particular eye.

After the laser procedure was finished the patients were taken under the microscope to finish up the surgery. The surgical procedure was completed by phacoemulsification. The Stellaris phacoemulsification machine (Bausch \& Lomb, New York, USA) was used in venturi fluidics mode. A standard intra ocular lens (IOL) was implanted in the capsular bag. The type of lens differed according to patient need and other variables (Photograph 3).

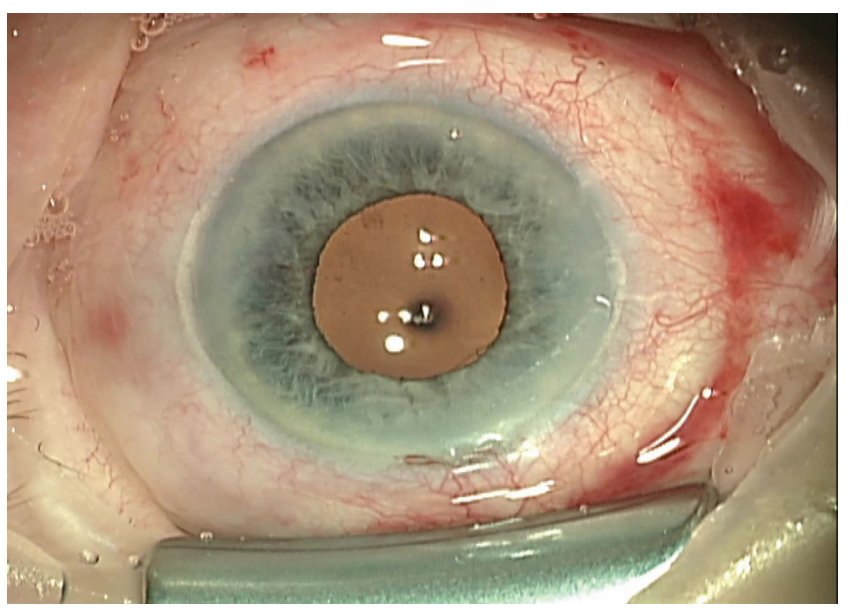

Photograph 3 showing, the completion of femtosecond assisted cataract surgery.

Post-operative management was done according to the hospital set standard protocol as in other femtosecond laser assisted cataract surgeries. All cases were discharged on the same day of surgery except when the cases required admission due to other systemic illnesses. Before discharge intraocular pressure measurement and uncorrected visual acuity measurement was carried out. Follow up was as for other routine femtosecond laser assisted cataract surgeries. All the relevant data were entered in to a specifically made proforma for the study. Statistical analysis was done using Social Package for Statistical Services 19 (SPSS 19). 
Results: Of thirty eyes included in the study, 19 eyes were of females accounting for $63.3 \%$ of total study subjects; while 11 eyes $(36.7 \%)$ were of males. The mean age of the patients was 74.8 years (std. deviation 6.5) ranging from 63 to 88 years. According to LOCS III, the mean nuclear sclerosis in all subjects was 3.9 (Std. deviation 0.8) ranging from grade three to five. Mean absolute phaco time (APT) in all study subjects was 0.19 seconds (Std. deviation 0.24 ) ranging from 0.00 seconds to 0.80 seconds, mean effective phaco time (EPT) in study subjects was 0.02 seconds (Std. deviation 0.04 ) ranging from 0.00 seconds to 0.20 seconds while mean phaco power (MPP) used for all cases was $9.26 \%$ (Std. deviation 8.24 ) it ranged from $0.00 \%$ to $25 \%$ (Table 1). Complications noted were anterior capsular tag in one case and posterior capsular rupture in one case.

Table 1: showing descriptive analysis of various parameters used in the study

\begin{tabular}{cccc}
\hline Variables & Minimum & Maximum & Mean (SD) \\
\hline $\begin{array}{c}\text { Age } \\
\text { (Years) }\end{array}$ & 63.00 & 88.00 & $74.83(6.56)$ \\
$\begin{array}{c}\text { LOCS III } \\
\text { (Grading) }\end{array}$ & 3.00 & 5.00 & $3.96(0.80)$ \\
APT & 00.00 & 0.80 & $0.19(0.24)$ \\
$\begin{array}{c}\text { Seconds) } \\
\text { EPT }\end{array}$ & 00.00 & 0.20 & $0.02(0.04)$ \\
$\begin{array}{c}\text { Seconds) } \\
\text { MPP }(\%)\end{array}$ & 00.00 & 25.00 & $9.26(8.24)$ \\
\hline
\end{tabular}

\section{Discussion:}

Femtosecond lasers and their application to cataract surgery constitute a major innovation in modern ophthalmic surgery. ${ }^{15}$ These lasers, which act on the near infrared wavelength $(1030 \mathrm{~nm})$ are capable of penetrating the transparent and even opaque cornea, with the only limitation of an eventual corneal vascularisation and the densely calcified plates. They are guided in their intraocular application by 3 dimensional image systems of the anatomy of the anterior segment of the eye. In this way they act in a very precise way, delivering energy to perform corneal incisions, capsulorhexis, softening or breaking the nucleus, thus enabling elimination of the cataract. The surgical procedure performed in this way, facilitates optimization of the surgical time, minimizing the surgeon's work (the least controllable variable of all those involved in cataract surgery) to the elimination of the cortical remains and the implantation of the intraocular lens. ${ }^{15}$
Taking into consideration that cataract surgery is the most commonly performed surgical procedure in Ophthalmology; it is not surprising that efforts are constantly made to improve its safety and efficacy. The shift from manual extracapsular cataract extraction to mechanized, ultrasound-assisted, small-incision cataract surgery has now been achieved, which has resulted in enormous benefits. ${ }^{16}$

Implementation of femtosecond laser in various types of cataract surgeries has resulted in excellent outcome. The changing trend of doing an aphaco or pauci phaco femtosecond laser assisted cataract surgeries in different grades of cataract too has been an area of focus. In an unpublished study the results of femtosecond laser use in cataract surgeries have shown to reduce EPT, APT and MPP remarkably when compared with standard phacoemulsification for cataract surgeries. ${ }^{17}$

In this study as well, it is clearly evident with the use of femtosecond laser for cataract surgery all the important parameters of phacoemulsification like APT, EPT, MPP have reduced drastically resulting in to minimal phaco energy used or otherwise known as pauci phaco.

More importantly getting a precise and circular capsulorhexis of 5.5/5 mm using femtosecond laser was very useful in completing the surgery, however a case did have remnant of anterior capsule as a tag where capsulorhexis was completed using Uttrata forceps later the case was then operated as other routine femtosecond laser assisted cases.

Before the advent of femtosecond laser there have been reports of using high mean phaco power (energy), APT, EPT while performing standard phacoemulsification with posterior chamber intraocular lens implantation. ${ }^{18}$ However, with use of femtosecond lasers softer forms of cataracts can be emulsified using no phaco energy and even hard brown cataracts can be emulsified by using minimal phaco energy as suggested by this study as well as the previous study. ${ }^{17}$

Minimal or no complications during femtosecond laser assisted cataract surgeries even in harder brown cataracts makes it even more desirable for the surgeon to use femtosecond laser for cataract surgery at present as this study suggested.

Recent researches suggest that the corneal endothelial cell loss is very less when compared to anyother types of cataract surgery when using femtosecond laser thus increasing its value more as corneal endothelial cell loss tends to increase with increase in MPP, APT and EPT. ${ }^{19}$

\section{Conclusions:}


This study concludes that, with the help of the laser, we are coming closer to the objective of removing the cataract nucleus with minimal or no requirement for phacoemulsification energy. With further innovation and optimization of technique and instruments, including laser dynamics and softening patterns, femtosecond laser-assisted cataract surgery will become phacoless ("aphaco") for most cataracts mainly softer ones, whereas brown/black or denser cataracts may still require the "pauci-phaco" technique.

\section{Acknowledgement:}

I would like to thank all the patients and their care takers for providing me support during the study. I would also like to pass my debt to University of Bochum, Department of Ophthalmology, for providing me an opportunity to conduct a research on femtosecond laser assisted cataract surgery in brown/hard cataracts.

\section{Conflict of interest: none}

\section{Funding: none}

\section{References:}

1. Semmens JB, Li J, Morlet N, et al. Trends in cataract surgery and postoperative endophthalmitis in Western Australia (1980 -1998): the Endophthalmitis Population Study of Western Australia. Clin Experiment Ophthalmol 2003;31:213-9.

2. ErieJC,BaratzKH,HodgeDO, etal.Incidenceofcataract surgery from 1980 through 2004: 25-year populationbased study. J Cataract Refract Surg 2007;33:1273-7.

3. Bali SJ, Hodge C, Lawless M, etal. Femtosecond Laser for Cataract Surgery. Ophthalmology 2012;119:891-9.

4. Ratkay TI, Juhasz T, Horvath C, et al. Ultra-short pulse (femtosecond) laser surgery: initial use in LASIK flap creation. Ophthalmol Clin North Am 2001;14:347-55.

5. Kezirian GM, Stonecipher KG. Comparison of the IntraLase femtosecond laser and mechanical keratomes for laser in situ keratomileusis. J Cataract Refract Surg 2004;30:804-11 .

6. GilCR, TeusMA, deBenitoLL, etal. Femtosecondlaser vsmechanicalmicrokeratome forhyperopiclaserinsitu keratomileusis. Am J Ophthalmol 2011;152:16 -21.

7. Sutton G, Hodge C. Accuracy and precision of LASIK flap thickness using the IntraLase femtosecond laser in 1000 consecutive cases. J Refract Surg 2008;24:802-6.
8. Kim P, Sutton GL, Rootman DS. Applications of the femtosecond laser in corneal refractive surgery. Curr Opin Ophthalmol 2011;22:238-44.

9. Sepehr F. Femtosecond Laser Cataract Surgery. J Ophthalmic Vis Res 2011;6:151.

10. Kranitz K, Agnes $\mathrm{T}$, Kata $\mathrm{M}$, et al. Femto second laser Capsulotomy and Manual Continuous Curvilinear Capsulorrhexis Parameters and Their Effects on Intraocular Lens centration. J Refract Surg 2011;27:558-563.

11. Majid M, Daniel SC, Maylon H. Femtosecond Laser-Assisted Cataract Surgery: A Current Review. Middle East Afr J Ophthalmol 2011;18:285-91.

12. Kothari K, Jain SS, Shah NJ. Anterior capsular staining with trypan blue for capsulorhexis in mature and hypermature cataracts. A preliminary study. Indian J Ophthalmol 2001;49:177-80.

13. Chylack LT, Wolfe JK, Singer DM, et al. The Lens Opacities Classification System III. The Longitudinal Study of Cataract Study Group. Arch Ophthalmol 1993;111:831-6.

14. The precision that changes everything: OptiMedicaCatalys review in. http://www.optimedica.com/ catalys-overview/

15. Jorge LA. Cataract surgery with femtosecond lasers. Saudi Journal of Ophthalmol 2011;25:219-23.

16. Rao SK, Jhanji V, Fan AH. Femtosecond Laser: Is It the Way Forward for Cataract Surgery? Asia-Pacific Journal of Ophthalmol 2012;1:3-4.

17. Conrad HI, Hengerer FH, Schultz T, et al. Effect of Femtosecond Laser Fragmentation of the Nucleus With Different Softening Grid Sizes on Effective Phaco Time in Cataract Surgery. J Cataract Refract Surg 2012;38:1888-94.

18. Ermiss SS, Ozturk F, Inan UU. Comparing the efficacy and safety of phacoemulsification in white mature and other types of senile cataracts. $\mathrm{Br} \mathrm{J}$ Ophthalmol 2003;87:1356-9.

19. Friedman NJ, Palanker DV, Schuele G, et al. Femtosecond laser capsulotomy. J Cataract Refract Surg 2011;37:1189-98. 